Int. J. Dev. Biol. 49: 873-879 (2005)

doi: $10.1387 / \mathrm{ijdb} .051986 \mathrm{da}$

Short Communication

\title{
Rab11 is required during Drosophila eye development
}

\author{
DEBASMITA P. ALONE, ANAND K. TIWARI, LOLITIKA MANDAL, MINGFA LI ${ }^{1}$, BERNARD M. MECHLER ${ }^{1}$ \\ and JAGAT K. ROY* \\ Cytogenetics Laboratory, Department of Zoology, Banaras Hindu University, Varanasi, India and \\ ${ }^{1}$ Department of Developmental Genetics, German Cancer Research Centre, Heidelberg, Germany
}

\begin{abstract}
In an effort to identify the role of Rab11, a small GTP binding protein, during Drosophila differentiation, phenotypic manifestations associated with different alleles of Rab11 were studied. The phenotypes ranged from eye-defects, bristle abnormalities and sterility to lethality during various developmental stages. In this paper, our focus is targeted on eye defects caused by Rab11 mutations. A novel P-element insertion in the Rab11 locus, Rab1 1 ${ }^{\text {mo }}$, displayed characteristic retinal anomalies, which could be reverted by P-element excision and expression of Rab11+ transgenes. During larval development, Rab11 is widely synthesized in photoreceptor cells and localizes to the rhabdomeres and lamina neuropil in adult eyes. Photoreceptors and associated bristles failed to be formed in homozygous clones generated in Rab11 ${ }^{E P(3) 3017}$ eyes. Decreased levels of Rab11 protein and increased cell death in $R_{a b 11^{m o}}$ third-instar larval eye-antennal discs suggest that the retinal defects originate during larval development. Our data indicate a requirement for Rab11 in ommatidial differentiation during Drosophila eye development.
\end{abstract}

KEY WORDS: Drosophila, Rab11, photoreceptor cell, rhabdomere

The regulation of intercellular communication is essential for proper development and survival of all multicellular organisms. This communication is brought about by well coordinated protein transport mechanisms which determine the route by which proteins and peptides are released into its surrounding micro-environment. Eukaryotic cells contain a highly dynamic set of membrane compartments that are responsible for packaging, sorting and recycling of bio-molecules whose transport is mediated by vesicles. Vesicle trafficking is regulated by specific players among which the Rab/Ypt family of proteins plays a major role. These proteins constitute the largest group within the Ras GTPase superfamily and are master regulators of vesicular transport in eukaryotic cells (Urbe et al., 1993; Zerial and McBride, 2001). Mammalian genomes contain more than 60 known Rab genes (Bock etal., 2001), while 29 Rabgenes have been so far identified in Drosophila (Pereira-Leal and Seabra, 2001). Each Rab protein is characterised by a distinctive localisation in the cell and works at a specific stage of vesicular transport pathway.

The vertebrate members of the Rab11 subfamily - Rab11a, Rab11band Rab25-have been reported as regulators of endocytic membrane recycling in both polarized and non polarized cells (Casanova et al., 1999; Cox et al., 2000; Wang et al., 2000) and are also involved in exocytosis (Chen et al., 1998; Urbe et al., 1993; Goldenring et al., 1994; Goldenring et al., 1996; Calhoun and Goldenring, 1997; Calhoun et al., 1998). These studies that were performed on unicellular organisms or isolated cultured cells provided insights into the molecular properties and subcellular localisation of Rab11 as well as on the underlying mechanism of vesicular transport. However, it is only recently that the role of Rab11 during development and differentiation has begun to be appreciated. Drosophila has a single Rab11 gene and studies have shown its requirement during oocyte polarization (Dollar et al., 2002; Jankovics et al., 2001), cytoskeletal remodeling (Riggs et al., 2003) and membrane morphogenesis (Pelissier et al., 2003; Zerial and McBride, 2001).

Due to precisely organized architecture, the Drosophila eye is an effective model for addressing questions related to processes including cell signaling, neuronal connectivity, control of cell proliferation and vesicular transport. Vesicular transport is undoubtedly critical during eye development. Over the last few years, studies of Drosophila Rab1 and Rab6 demonstrated the role of these proteins as important players in processing and/or transport of rhodopsins (Satoh et al., 1997, 2005; Shetty et al., 1998). In this communication, we show that Rab11 is one of the key players during Drosophila eye development.

\section{Results and Discussion}

\section{Punctate Rab11 staining in eye imaginal discs}

As the C-terminal domains of Rab proteins contain specific motifs directing vesicle targeting (Chavrier et al., 1991), we generated Rab11 polyclonal antibodies against a C-terminal 27-

\footnotetext{
*Address correspondence to: Dr. Jagat K. Roy. Cytogenetics Laboratory, Department of Zoology, Banaras Hindu University, Varanasi 221 005, India. Fax: +91-542-236-8457. e-mail: jkroy@bhu.ac.in
} 

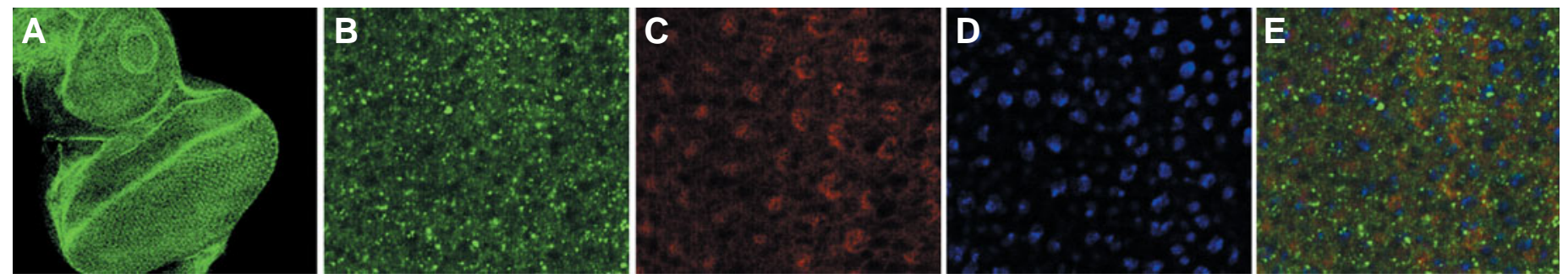

Fig. 1. Distribution of Rab11 on third-instar eye-antennal discs. (A) The Rab11 protein is widely distributed in all ommatidial and antennal cells. (B) Higher magnification of the ommatidial cells reveals a puntate staining. (C, D) Counterstaining of the immunostained eye disc with Rhodamine-Phalloidin and DAPI, which detect F-actin and DNA, respectively. (E) Merged view of (B, C and D) showing the cytoplasmic localisation of Rab11 and a partial overlap with F-actin.

mer synthetic peptide. These antibodies were affinity purified and used for localising Rab11 in tissues. Western blot of proteins extracted from various developmental stages showed a single polypeptide of $23 \mathrm{kDa}$ corresponding to the expected molecular mass of Rab11. Immunostaining on eye-antennal discs from third-instar larvae revealed that Rab11 is distributed in the ommatidial precursor cells and in the antennal cells (Fig. 1A). Higher magnification (Fig. 1B) reveals a punctate staining pattern characteristic of a protein involved in vesicular transport. Counterstaining was done with Phalloidin, which specifically stains polymerised actin or F-actin (Fig. 1C) and DAPI for DNA (Fig. 1D). As shown in Fig. 1E, Rab11 predominantly localises in the cytoplasm and occasionally overlaps with F-actin. The punctate staining indicates association with membrane organelles. Specificity of the antibodies was confirmed by competing antibody binding with the 27-mer synthetic peptide. When the eye-antennal discs were immunostained with an overnight pre-incubated mixture of anti-tubulin and anti-Rab11 antibodies, the expected patterns of staining were observed (Fig. 2 A,B). However, when the discs were immunostained with a pre-incubated mixture of anti-tubulin and anti-Rab11 antibodies in the presence of the 27mer synthetic peptide, no Rab11 staining could be detected (Fig.
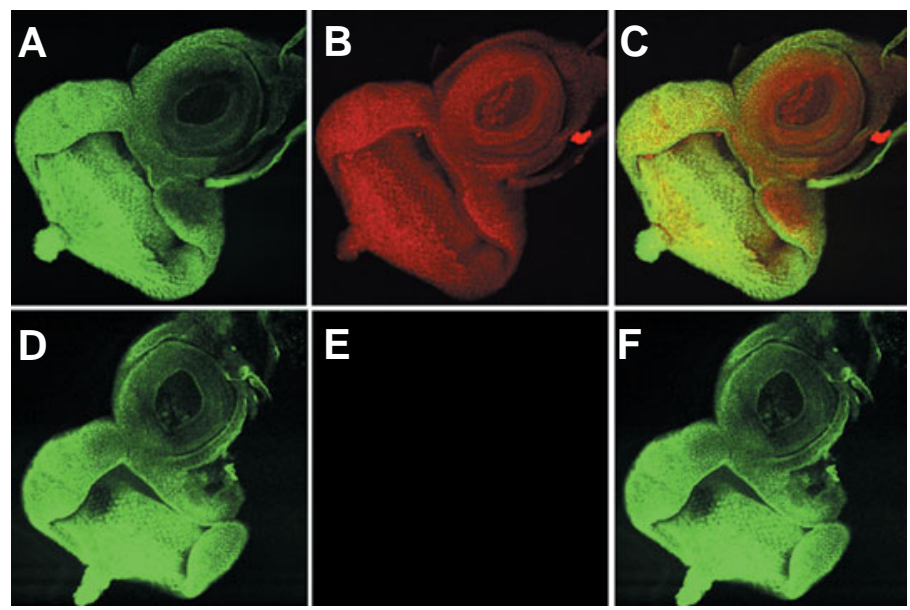

$2 \mathrm{E})$; although the discs showed expected pattern of staining for tubulin (Fig. 2D). Immunostaining on adult head sections revealed a strong distribution of Rab11 in the rhabdomeres, lamina neuropil and photoreceptor terminals (Fig. 3A) whereas no Rab11 could be detected in the medulla and lobula (Fig. 3B). All together, the localisation of Rab11 in the developing eyes indicates that this protein may play a critical role during the development of this organ in larval or pupal stages. On this basis, we investigated the requirement of Rab11 during eye development in Drosophila.

Rab11 is essential for viability of photoreceptors and bristles

Since the mutant animals for the two available alleles of Rab11, namely, $R a b 11^{1 /(3) j 2 D 1}$ and $R a b 11^{E P(3) 3017}$ - die as embryos and early larvae, we generated $R a b 11^{1 /(3) j 2 D 1}$ somatic clones using EGUF/hid method (Stowers and Schwarz, 1999) and analysed their phenotype in adult eyes. We found that all the FRT$R a b 11^{1 /(3) j 2 D 1}$ clones displayed normal ommatidia (Fig. 4B). Scanning electron microscopic analysis of a representative homozygous clone revealed wild type eye architecture (Fig. 4E, H). Since Rab1 1/(3)j2D1 results from a P-element insertion into the second intron of $R a b 11$, it is plausible that the insert may specifically affect early development and viability but may not be essential for the eye development. To circumvent the absence of an eye phenotype we used the $R a b 11^{E P(3) 3017}$ to generate retinal clones. In homozygous FRT-Rab11EP(3)3017 clones we found that the photoreceptor cells and associated bristles were absent indicating that the mutation induced cell lethality (Fig. 4C). Scanning electron microscopic studies of a representative homozygous clone revealed the absence of photoreceptor cells and bristles (Fig. 4F, I). We concluded that normal function of Rab11 is essential for cell

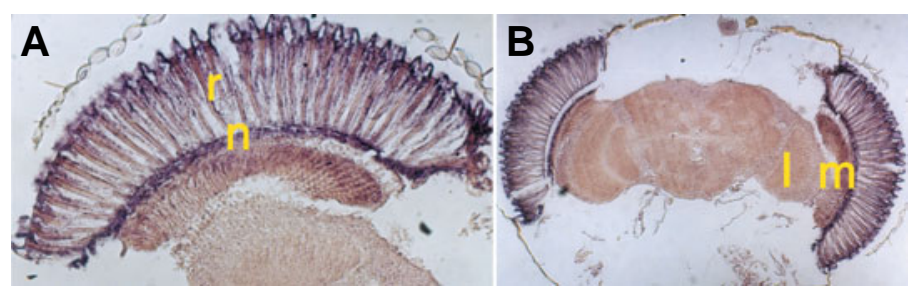

Fig. 2 (Left). Specificity of the Rab11 antibody. (A-C) Eye-antennal disc stained with anti-tubulin and anti-Rab11 antibodies (control) showing normal pattern of distribution of tubulin (A) and Rab11 (B) proteins. (D-F) Eye-antennal disc stained with a pre-incubated mixture of anti-tubulin, anti-Rab11 antibodies. and the 27-mer synthetic peptide used for generating the rabbit anti-Rab11 antibodies, showing normal staining for tubulin (D) and absence of staining for Rab11 (E). (C) and (F) are merged pictures of $(A, B)$ and $(D, E)$ respectively.

Fig. 3 (Right). Rab11 distribution in adult head and eye sections. (A) Rab11 is detected in the photoreceptors, primarily in the rhabdomeres ( $r$ ) and the lamina neuropil (n). (B) No significant Rab11 staining could be detected in medulla ( $m$ ) and lobula (I). (A) is an enlargement of eye shown to the right in (B). 
viability in eyes. In order to complement the observations made with $R a b 11^{E P(3) 3017}$, it was desirable to have an adult viable allele of Rab11, which could be directly used for such phenotypic studies. One such insertion was recovered during a P-element mutagenesis screen in our laboratory and was utilized for this study.

\section{Ommatidia defects linked to P-insertion in the Rab11 gene}

By mobilizing a P-element in a nearby locus, we generated an insertion in Rab11 gene. In-situlocalization of this P-element on the polytene chromosomes mapped the insertion to 93B region. Sequence analysis of a $1.9 \mathrm{~kb}(1.1 \mathrm{~kb}$ and $0.8 \mathrm{~kb})$ plasmid rescued fragments revealed a P-element insertion in the 5' regulatory region of the Rab11 gene (Fig. 5). This mutation produced viable adults. The mutant animals displayed low viability and some homozygotes survived only till larval, pupal or pharate adult stages. The pharate adults as well as adults showed dark patches in the ommatidia, which upon close examination proved to be malformed ommatidia, thus this allele was named as $R a b 11^{m o}$. $R a b 11^{1 /(3) j 2 D 1}$, an insertion in second intron and $R a b 11^{E P(3) 3017}$, an insertion in second exon of $R a b 11$ gene die as embryos and during first instar stage of development, respectively (Fig. 5). Rab1 $11^{m o}$ ho-
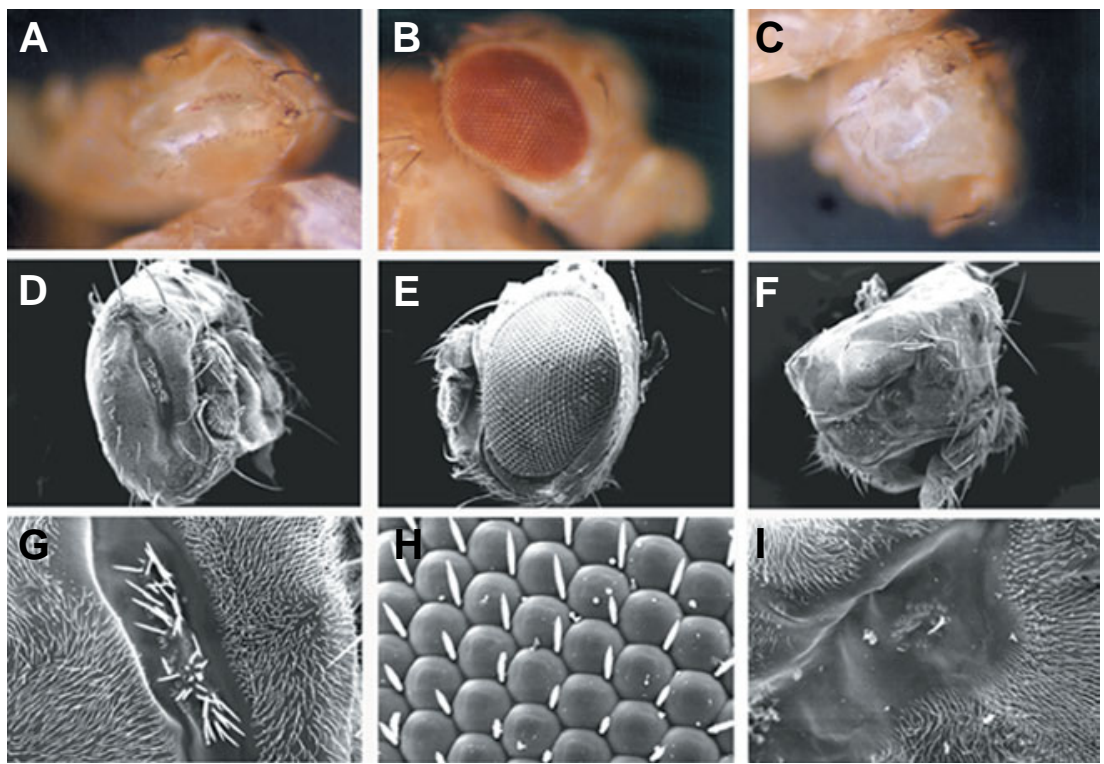

Fig. 4. Light and scanning electron microscopic analysis of Rab11 mutant somatic clones. (A,D,G) In yw; EGUF/+; FRT82B GMR-hid3R CL/FRT82B GMR-hid3R CL eyes photoreceptor cells were destroyed due to the induction of cell death by GMR-hid. (B,E,H) In yw; EGUF/+; FRT82BGMR-hid3R CL3R/FRT82B Rab11/(3)/2D1 the eye organization is similar to that of wild type showing a regular pattern in the ommatidial distribution. (C,F,I) In yw; EGUF/+; FRT82B GMR-hid3R CL3R/FRT82BRab11EP(3)3017 eyes all photoreceptor cells are missing indicating that Rab11 is required for photoreceptor cell differentiation and viability. mozygotes were also sterile and showed antennal bristle abnormalities, phenotypes that have been noted in other Rab11 alleles (Abdelilah-Seyfried et al., 2000; Jankovics et al., 2001; Dollar et al., 2002).

To determine whether the $\mathrm{P}$-insert in Rab11 is responsible for these defects we carried out excision of the P-element in Rab1 1 mo and generated revertants. Out of 53 revertants, one pure excision line exhibiting a wild type phenotype was obtained. This indicates that the phenotype/s were only due to the Rab11 mutation and no second site mutation was responsible. To further test this conclusion, we constructed pCasper-Rab11 transgene and following transformation we obtained two independent Rab11 transgenic lines (pCasper-Rab11a\& pCasper-Rab11b). Enhanced level of Rab11 protein of the expected size was obtained on Western blots of protein extracts from Rab11transgenic larvae in comparison to wild type, hence indicating them to be functional transgenes. The pCasper-Rab11a; Rab11mo/Rab11mo flies showed nearly wild type eye morphology indicating that the mutant phenotype resulted from a mutation in Rab11. Apart from the eye defects, the transgene also abolished all other mutant phenotypes including antennal bristle abnormalities and sterility. We even found that a single copy of the Rab11 transgene could restore viability of $R a b 11^{E P(3) 3017}$ and Rab1 1/(3)/2D1 homozygous animals.

In this communication, we particularly focused our attention on the eye defects produced by Rab1 1 mo. Numerous dark patches, which were variable in size, could be detected in Rab1 1 mo $^{\mathrm{mo} e s}$ (Fig. 6B) and showed no site- specificity. Some eyes had only 2-3 small isolated dark patches while others contained hardly any normal ommatidia. A representative wild type eye is compared with the mutant eye in Fig. 6 A,C, respectively. Adult escapers of $R a b 11^{\mathrm{mo}} / R_{a b 1}$ EP(3)/3017 transheterozygotes, which were primarily larval lethal, also showed eye defects while the eyes of $R a b 11^{m o} / R a b 11^{1 /(3) / 2 D 1}$ individuals were normal with no obvious defects (Fig. 6D).

Scanning electron microscopic (SEM) studies revealed that the dark patches were made of deformed ommatidial cells. In comparison to the wild type regular ommatidial arrangement (Fig. $6 \mathrm{E}, \mathrm{I})$, fused ommatidia and missing bristles were detected in

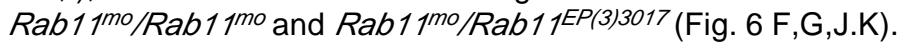
No alteration could be detected in $R a b 11^{m o} / R a b 11^{1 / 3) j 2 D 1}$ (Fig. 6 $\mathrm{H}, \mathrm{L})$. We wondered if the dark patches, fused ommatidia and missing bristles correspond to something deep within the ommatidia.

Histology contributed to our understanding of the nature of

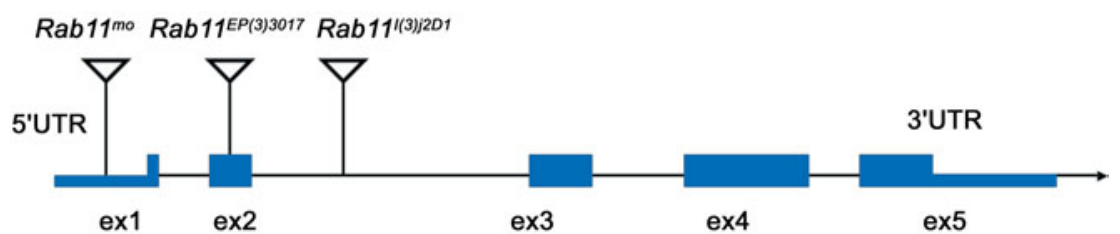

Fig. 5. Schematic representation depicting the position of P-element insertions in Rab11 mutants. The exons have been represented as 'ex' and the introns are shown as thin lines. The $5^{\prime}$ UTR and $3^{\prime}$ UTR are marked. Rab11 $11^{\mathrm{mo}}$ is an insertion in the $5^{\prime}$ regulatory region, Rab11/(3)j2D1 is an insertion in the second intron and Rab11EP(3)3017 in the second exon of Rab11 gene, respectively. 

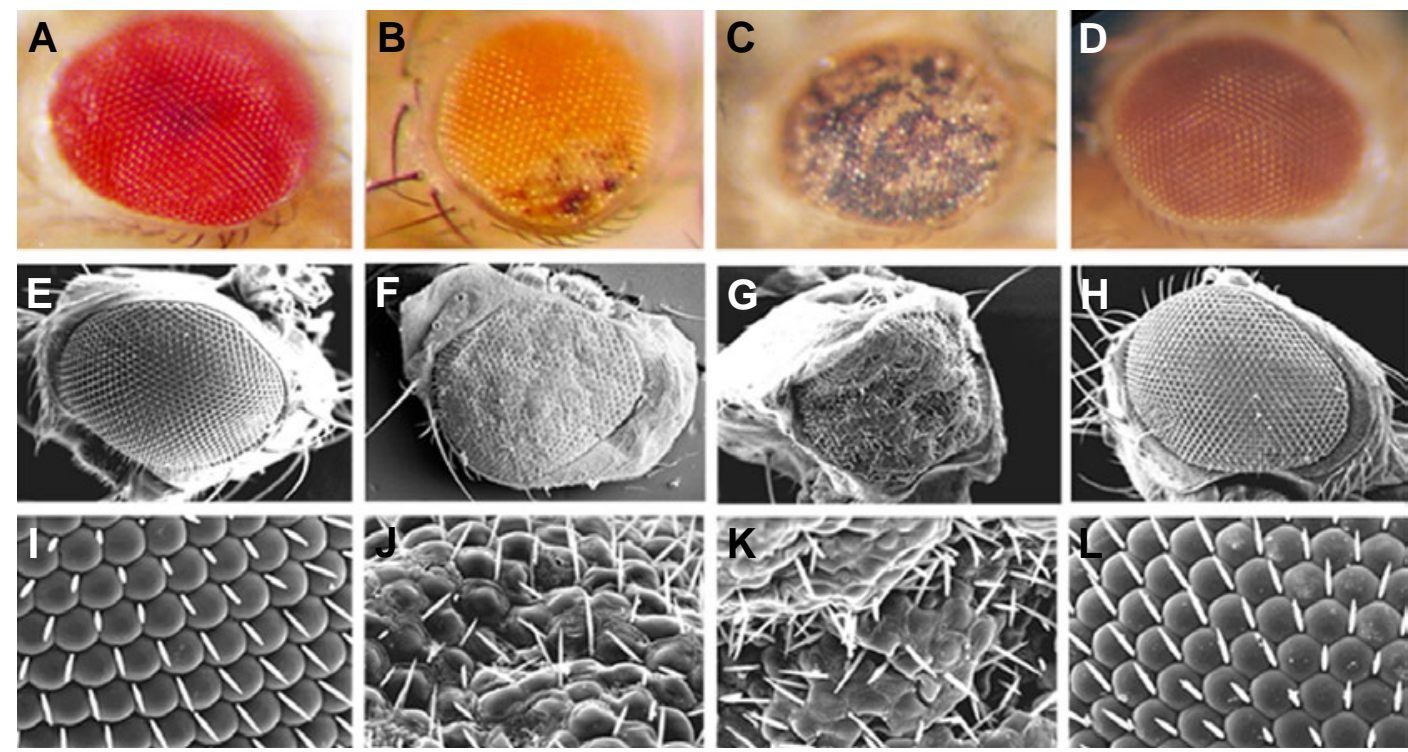

Fig. 6. Eye defects associated with $\mathbf{R a b 1 1}$ alleles. (A,E,I) Eyes from wild type flies showing an organised ommatidial architecture. (B, $\mathbf{F}, \mathbf{J})$ and $(\mathbf{C}, \mathbf{G}, \mathbf{K})$ Eyes of Rab1 $11^{\mathrm{mo}} /$ Rab1 $11^{\mathrm{mo}}$ showing abnormalities from minor $(\mathbf{B})$ to greater severity (C). (F,G,J,K) SEM pictures show fusion of ommatidia and missing bristles in mutant individuals Rab11mo/Rab11EP(3)3017 adult esacpers also displayed retinal defects. (D,H,L) Eyes of Rab11mo/Rab11/(3)j2D1 flies showed no obvious defect and were similar to wild type.

retinal anomalies. Toluidine blue stained sections of adult eyes from flies of different genotypes were examined. In comparison to the wild type regular ommatidial arrangement (Fig. 7A), photoreceptor cells could be hardly seen in the severely affected Rab1 $1^{\mathrm{mo}}$ / Rab $11^{m o}$ mutant eyes (Fig. 7B). In these individuals, only residual cell fragments appeared to be present in the retina. The drastic loss of retinal structures could be related to the frequent collapse of the eyes noticed during critical point drying for SEM studies. Eye sections of the Rab11 revertant and of transgenic pCasperRab11a; Rab1 1mo/Rab1 1mo flies displayed a regular ommatidial arrangement and preservation of the photoreceptor rhabdomere structure (Fig. 7 C,D).

Since a single Rab11 transgene could abolish the defects associated with the Rab1 $1^{m o}$ mutations, it is possible to envisage

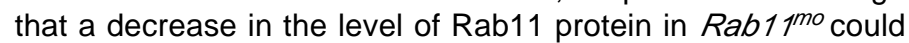
lead to retinal defects. A comparison of the amount of Rab11 proteins between wild type and Rab1 $1^{\text {mo }}$ flies revealed only a decrease of $10-50 \%$ in mutant individuals (Fig. 8A). Similarly, the body extracts showed $20 \%$ decrease in Rab11 level in the mutants. In contrast, when fly heads were separated from the rest of the body, we noticed an eight-fold decrease in Rab11 protein discs (Fig. 8C) in comparison to that in wild type discs (Fig. 8B) even though the number of ommatidial cells were similar to wild type individuals (as seen by Elav antibody staining in the later section), indicating that a decrease in Rab11 protein during third-instar larval development leads to retinal defects in adults.

\section{Retinal deformities originate during late larval development}

In Drosophila eye, cell differentiation begins with the progression of the morphogenetic furrow across the field of progenitor cells, such that cells at the furrow are just beginning to differentiate, whereas those situated more posteriorly are progressively developing (Ready et al., 1976). Immuno-fluorescence staining of the Elav protein in third-instar larval eye discs revealed an abnormal ommatidial arrangement in Rab1 1 mo/Rab 1 1mo $^{\mathrm{mo}}$ (Fig. 9A, B). Similarly, immunostaining with the monoclonal anti-Mab22C10 antibody showed disorganised bundles of axons between the brain and the eye discs in $R a b 11^{m o}$ (Fig. 9C, D). Finally acridine orange staining revealed an increased number of dying cells below the morphogenetic furrow in $R a b 11^{m o}$ by comparison to wild-type (Fig. 9E, F). Taken together, these data suggest that the retinal defects could be traced to ommatidial differentiation which
Fig. 7. Toluidine blue stained retinal sections (A) Horizontal section of a wild type fly head showing the regular arrangement of ommatidial cells. (B) Horizontal

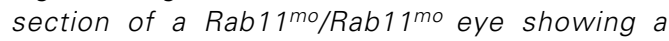
disorganised ommatidial cell arrangement. Note the severe loss of retinal structures. (C) Horizontal section of a Rab11 revertant eye showing the regular wild type ommatidial and (D) horizontal sections of PcasperRab11a; Rab11mo/Rab11mo flies showing a normal ommatidial arrangement.
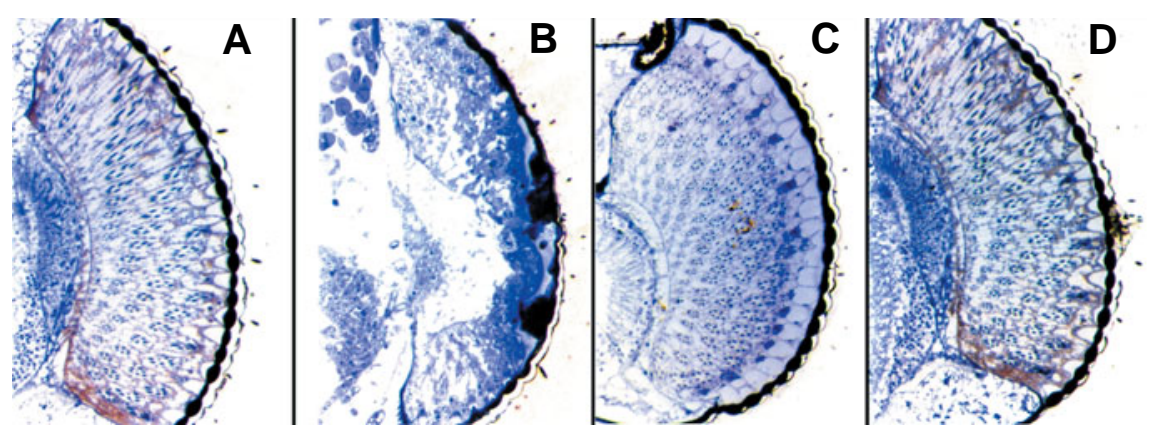

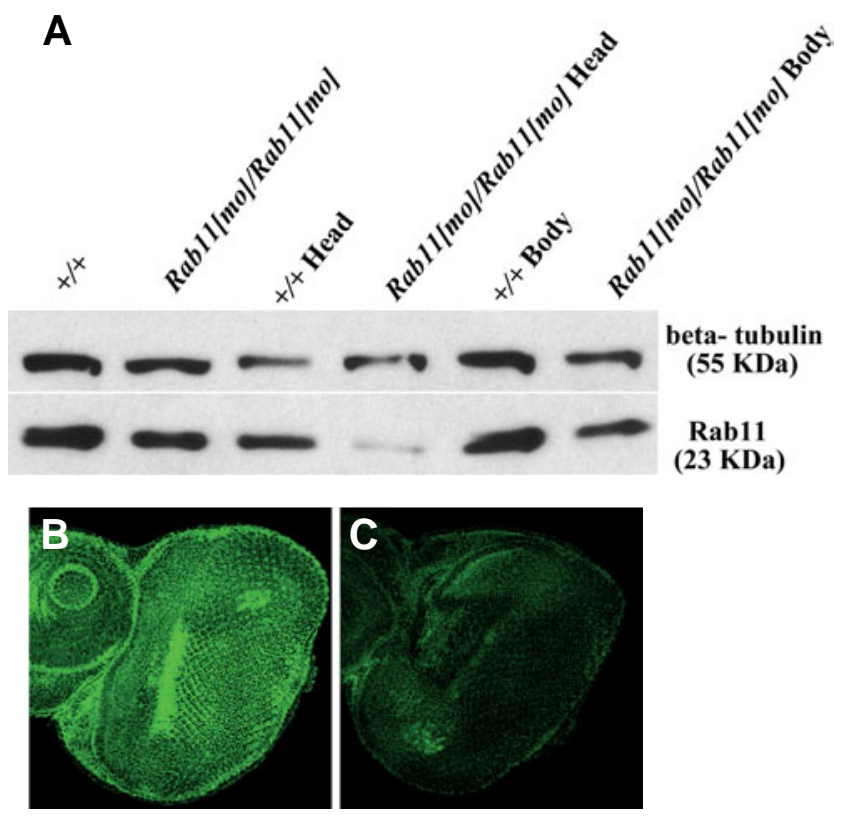

Fig. 8. Levels of Rab11 protein in $R a b 11^{m o} / R_{a b 11}$ mo tissues. (A) Western analysis of adult extracts showing a decrease in Rab11 protein in Rab11mo/Rab11mo individuals in comparison to wild type. Note the pronounced decrease of Rab11 level in head in comparison to body. Control loading was performed by comparing the amount of beta-tubulin. (B) Immuno-staining of Rab11 protein in wild type and (C) Rab11 mo/Rab11 mo third-instar larval discs. A decrease in the intensity of of Rab11 staining is less intense in (C) compared to (B).

takes place during late larval development and to the death of numerous neuronal cells during late third instar and early pupal stages. As ommatidial morphogenesis is a complicated process, inadequate membrane growth and recruitment, as well as anomaly in rhodopsin transport due to altered Rab11 protein may lead to eye degeneration in mutant eye discs. In support of our conclusion, a very recent article also describes the requirement of Rab11 for the transport of rhodopsin in Drosophila photoreceptor cells (Satoh et al., 2005)

In summary, our results demonstrated the role of Drosophila Rab11 during eye morphogenesis. We showed that the Rab1 1 mo P-element insertion induce retinal deformities which could be abolished with a Rab11 transgene. Precise excision of the Pelement from Rab11mo also leads to reversion of the mutant phenotype. We found that a reduced level of Rab11 protein is associated with cell death in Rab1/mo eye-antennal discs and show that these retinal defects originate during larval development. Our findings reveal that Rab11 is a key player during ommatidial differentiation in Drosophila.

\section{Materials and Methods}

\section{Fly stocks}

Rab11EP(3)3017/TM6B (Abdelilah-Seyfried et al., 2000) and Rab1 1/(3)j2D1/TM6B (Bloomington Stock Centre) are existing P. insertions in the Rab11 gene. Rab11mo/TM6B is a P-element mutation generated in our lab and caries a P-element inserted in the 5 ' regulatory region of Rab11gene. $y w ; \Delta 2-3, S b / T M 6 B$ (JSK17, Robertson et al., 1988) and $y w ;$ TM3, Sb/TM6B (JSK-3, Bier et al., 1989) were the strains used for P-element mobilization. Pelement mobilization was done essentially as described by Cooley et al. (1988). The P\{ry+t7.2=neoFRT\} $82 B$ line (Xu and Rubin, 1993) was used for generating a third chromosome FRT-Rab11. These FRT-Rab11 and yw; EGUF/EGUF; FRT GMR-hid 3R CL/ TM2 (Stowers and Schwarz, 1999) were used for producing eye specific somatic clones. All flies were reared on standard yeast supplemented food at $22 \pm 1^{\circ} \mathrm{C}$.

\section{Antibodies}

A 27 amino acid synthetic peptide (CEGDVIRPSNVEPIDVKP TVTADVRKQ) corresponding to the C-terminal sequence of Rab11 was coupled to maleimide activated Keyhole Limpet Haemocyanin (KLH) and injected into rabbits for raising polyclonal antibodies. The cysteine residue was introduced as the first residue to facilitate tagging of the peptide to $\mathrm{KLH}$. The antibodies were purified using Protein-A agarose column and the absorbance at $280 \mathrm{~nm}$ for each fraction was determined. The antibodies were then purified using a sulpholink-27-mer-peptide column. To obtain the desired working titre of anti-Rab11 antibodies, different antibody dilutions were subjected to western blot analysis using total proteins extracted from different stages of fly development. Dilutions of 1:20,000 for western blot analysis and 1:200 for
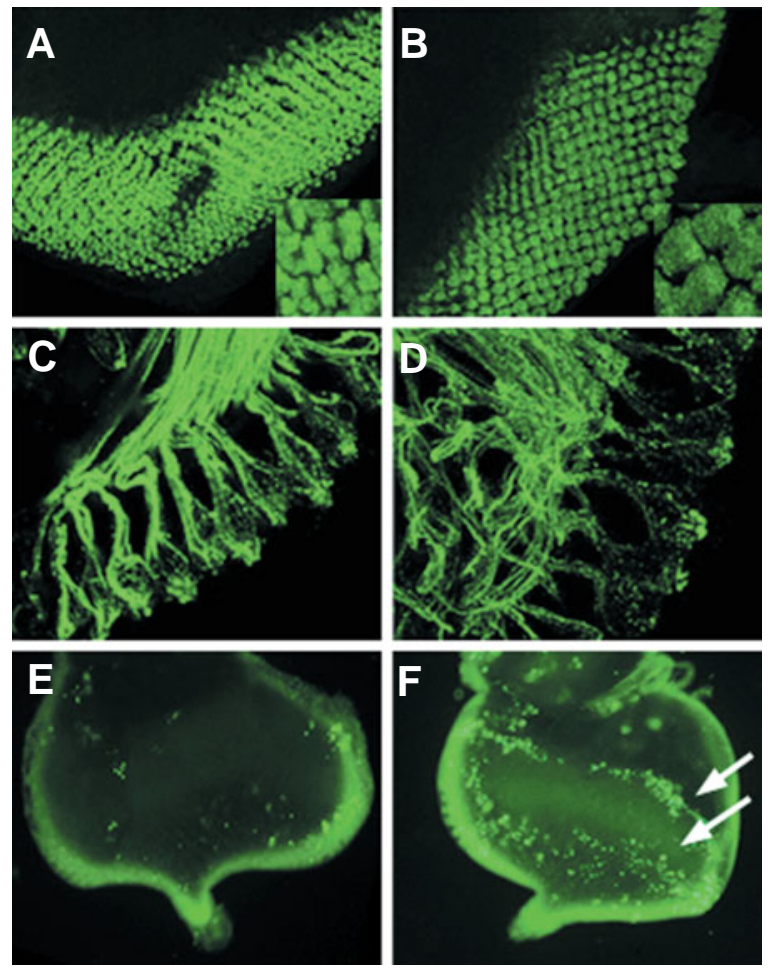

Fig. 9. Organisation and cell death in wild-type and $R a b 11^{m o} / R_{a b 1}{ }^{m o}$

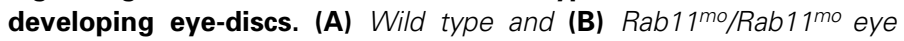
imaginal discs of late third instar larvae stained for the Elav protein, showing the ommatidial organisation. Insets display enlargements of single ommatidium. (C) Ordered axonal connection between the brain and eye disc in wild type and (D) disorganized bundles of axons in Rab11 mo/ Rab11mo larvae as seen by immunostaining with the mouse monoclonal $22 \mathrm{C10}$ antibody. Detection of cell death by Acridine Orange staining in (E) wild type and (F) Rab11mo/Rab11mo eye imaginal discs. An increased number of dying cells is detected posterior to the morphogenetic furrow in the mutant disc (arrow). 
immunostaining on intact tissues were routinely used.

\section{Rab11 clonal analysis}

Homozygous somatic clones of Rab11alleles were specifically induced in the eyes using the EGUF/hid technique. The Rab11 alleles, $R a b 11^{E P(3) 3017}$ and $R a b 11^{1 /(3) / 2 D 1}$ were recombined to the $P\{r y+t 7.2=n e o$ FRT $\} 82 B$ chromosome. The FRT82B Rab11 recombined arm was selected on the basis of neomycin resistance conferred by the FRT construct and the mutant phenotype due to the presence of P-element marker $w+$. Individual FRT flies were crossed with $y$ w; EGUF/EUGF; FRT GMR-hid 3R CL/TM2flies. Eye clones were examined in $y w$; EGUF/t; FRT82B Rab11 allele/ FRT GMR-hid $3 R C L$ males. Adult heads were dissected and subjected for histological and electron microscopic examinations.

\section{Generation of Rab11 ${ }^{\mathrm{mo}}$ and revertant}

A P-element insertional mutagenesis screen was initiated in the $93 \mathrm{~B}$ region using a $p L a c w+$ insertion line in $m v / g e n e$ (Bier et al., 1989). A series of insertions were generated and phenotypically analysed. One of the insertion mutant line showed abnormal ommatidia. A congenic line of the insertion was generated by crossing the flies for several generations to $w^{1118}$ eliminating background mutations. The resulting insertion was adult viable and displayed abnormal ommatidia. In-situ localisation mapped the P-element to chromosomal region 93B8-13. In order to identify the gene in which P-element was inserted in this line, the genomic DNA was digested by EcoR1 and standard plasmid rescue protocol (Pirrotta. 1986) was followed. It is expected that the plasmid DNA thus obtained will contain 3' region of $\mathrm{P}$-element having AmpRgene, ori, polylinker 2 (containing a Pst1 site) and the flanking genomic DNA. With the aim to eliminate the portion of P-element from the rescued genomic DNA, the plasmid DNA was digested with EcoR1 and Pst1 and three fragments of 1.9, 1.1 and $0.8 \mathrm{~kb}$ were obtained. Subsequent analysis revealed that 1.1 and $0.8 \mathrm{~kb}$ fragments were from genomic DNA almost devoid of $\mathrm{P}$-element and hence they were used for sequencing and cloning experiments. Sequence analysis of the plasmid rescued fragments showed that the P-element was inserted in Rab11, hence we named the new mutation under the designation Rab1 $1^{\text {mo }}$ [mo = malformed ommatidia].

\section{Cloning of the Rab11 gene and construction of a Rab11+ transgene}

A $\lambda$ FIXII Drosophilagenomic library (Stratagene, La Jolla, CA) was screened using either a labeled $1.1 \mathrm{~kb}$ plasmid rescued genomic DNA fragment or a PCR amplified 661bp from a Rab11 cDNA (EST GH10576) as probes. A $6.3 \mathrm{~kb}$ Xhol-Kpnl genomic DNA fragment containing the entire Rab11gene was cloned into the pCasper4 vector (Pirrotta, 1988). Transgenic flies were generated by microinjecting pCasper4-Rab11 DNA into $w^{1118} ; \Delta 2-3$, $S b /+$ flies according to standard protocol (Rubin and Spradling, 1982; Spradling and Rubin, 1982). Two out of six transgenic lines with an insertion in the second chromosome and producing wild type Rab11 protein were named as pCasper-Rab11aand pCasperRab11b. These two lines were individually crossed with the

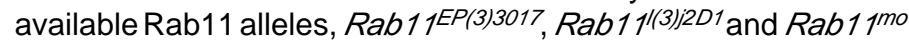
which were examined for developmental rescue in mutant homozygous and in trans-heterozygous conditions.

\section{Immunostaining, scanning electron microscopy and tolui- dine blue staining}

Whole organ staining was carried out as described by Patel, 1994 and immunostaining of paraffin embedded adult head sections was done according to Ausubel et. al., 1994. Plastic sections of adult eye were stained in a $1 \%$ toluidine-1\% borate aqueous solution for 2 min at $60^{\circ} \mathrm{C}$ (Cagan and Ready, 1989). The slides were rinsed in water to remove excessive stain and examined under light microscope. For SEM studies, adult flies of desired genotypes were decapitated, the proboscis was removed and both eyes were separated with the help of a sharp razor to facilitate accessibility of internal head structures for proper fixation and mounting in the appropriate orientation. Samples were fixed in $2.5 \%$ gluteraldehyde for overnight at $4^{\circ} \mathrm{C}$, washed $3 \times 30$ min with $0.1 \mathrm{M}$ PBS, dehydrated in ascending acetone grades and then critical point dried. They were then mounted on studs in desired orientation under the stereo-binocular microscope and coated with gold (thickness 30 to $35 \mathrm{~nm}$ ). Scanning was done on SEM mode in a LEO 435VP electron microscope at $15 \mathrm{kV}$.

\section{Western analysis}

Ten adult heads of the desired genotype were dissected in PBS, transferred to a microfuge tube containing $30 \mu$ protein sample buffer (100mM Tris, pH6.8, 1M DTT, 10\% SDS, 100 mM PMSF, pH $6.8,1 \%$ bromophenol blue and $1 \%$ glycerol) and boiled for $10 \mathrm{~min}$ followed by quick chilling and centrifugation at $5000 \mathrm{rpm}$ for $10 \mathrm{~min}$ at $4^{\circ} \mathrm{C}$. Proteins in the supernatant were separated by SDS-PAGE (12.5\%) and blotted on Immobilon-P membrane (Millipore). The blot was first incubated with rabbit anti-Rab11 polyclonal antibodies (dilution 1: 20,000) and then with a mouse anti- $\beta$ tubulin monoclonal antibody (dilution 1:50). The blots were developed using horseradish peroxidase labeled anti-rabbit and anti-mouse antibodies in conjunction with the ECL detection system (Amersham Pharmacia Biotech, UK) and quantitation was done with NIH Image J software.

\section{Acridine Orange staining}

Eye discs from the late third instar larvae were dissected out in Poels' Salt solution (PSS, Lakhotia and Tapadia, 1998), incubated in $1 \mu \mathrm{g} / \mathrm{ml}$ acridine orange (Sigma) solution in PSS for $3 \mathrm{~min}$, washed three times, mounted in PSS and immediately observed under a fluorescence microscope (Nikon E800) using a B-2A filter.

\section{Acknowledgements}

We greatly acknowledge the help of /stvan Török in the cloning of Rab11 gene and Howard $A$. Nash for his useful comments on the manuscript. The help of the Electron Microscopy Facility, AIIMS, New Delhi and Developmental Studies Hybridoma Bank, lowa for Elav and Mab22C10 antibodies is duly acknowledged. The work was supported by a grant from the Department of Science \& Technology, New Delhi and fellowships from the German Cancer Research Centre, Heidelberg, the Department of Biotechnology, New Delhi to JKR; and the Council of Scientific and Industrial Research, New Delhi to DPA.

\section{References}

ABDELILAH-SEYFRIED, S., CHAN, Y.M., ZENG, C., JUSTICE, N.J., YOUNGERSHEPHERD, S., SHARP, L.E., BARBEL, S., MEADOWS, S.A., JAN, L.Y. and JAN, Y.N. (2000). A gain-of-function screen for genes that affect the development of the Drosophila adult external sensory organ. Genetics 155:733-752. 
BIER, E., VAESSIN, H., SHEPHERD, S., LEE, K., MCCALL, K., BARBEL, S., ACKERMAN, L., CARRETTO, R., UEMURA, T., GRELL, E., JAN, L.Y. and JAN, Y.N. (1989). Searching for pattern and mutation in the Drosophila genome with a P-lacZ vector. Genes Dev. 3:1273-1287.

BOCK, J.B., MATERN, H.T., PEDEN, A.A. and SCHELLER, R.H. (2001). A genomic perspective on membrane compartment organization. Nature 409:839-841.

CAGAN, R.L. and READY, D.F. (1989). The emergence of order in the Drosophila pupal retina. Dev. Biol. 136:346-362.

CALHOUN, B.C. and GOLDENRING, J.R. (1997). Two Rab proteins, vesicleassociated membrane protein 2 (VAMP-2) and secretory carrier membrane proteins (SCAMPs), are present on immunoisolated parietal cell tubulovesicles. Biochem. J. 325:559-564.

CALHOUN, B.C., LAPIERRE, L.A., CHEW, C.S. and GOLDENRING, J.R. (1998). Rab11a redistributes to apical secretory canaliculus during stimulation of gastric parietal cells. Am. J. Physiol. 275:C163-170.

CASANOVA, J.E., WANG, X., KUMAR, R., BHARTUR, S.G., NAVARRE, J., WOODRUM, J.E., ALTSCHULER, Y., RAY, G.S. and GOLDENRING, J.R. (1999). Association of Rab25 and Rab11a with the apical recycling system of polarized Madin-Darby canine kidney cells. Mol. Biol. Cell 10:47-61.

CHAVRIER, P., GORVEL, J.P., STELZER, E., SIMONS, K., GRUENBERG, J. and ZERIAL, M. (1991). Hypervariable C-terminal domain of rab proteins acts as a targeting signal. Nature 353:769-772.

CHEN, W., FENG, Y., CHEN, D. and WANDINGER-NESS, A. (1998). Rab11 is required for trans-golgi network-to-plasma membrane transport and a preferential target for GDP dissociation inhibitor. Mol. Biol. Cell 9:3241-3257.

COOLEY, L., KELLEY, R. and SPRADLING, A. (1988). Insertional mutagenesis of the Drosophila genome with single P elements. Science 239:1121-1128.

COX, D., LEE, D.J., DALE, B.M., CALAFAT, J. and GREENBERG, S. (2000). A Rab11-containing rapidly recycling compartment in macrophages that promotes phagocytosis. Proc. Natl. Acad. Sci. USA 97:680-685.

DOLLAR, G., STRUCKHOFF, E., MICHAUD, J. and COHEN, R.S. (2002). Rab11 polarization of the Drosophila oocyte: a novel link between membrane trafficking, microtubule organization and oskar mRNA localization and translation. Development 129:517-526.

GOLDENRING, J.R., SMITH, J., VAUGHAN, H.D., CAMERON, P., HAWKINS, W. and NAVARRE, J. (1996). Rab11 is an apically located small GTP-binding protein in epithelial tissues. Am. J. Physiol. 270: G515-525.

GOLDENRING, J.R., SOROKA, C.J., SHEN, K.R., TANG, L.H., RODRIGUEZ, W., VAUGHAN, H.D., STOCH, S.A. and MODLIN, I.M. (1994). Enrichment of rab11, a small GTP-binding protein, in gastric parietal cells. Am JPhysiol267:G187-194.

JANKOVICS, F., SINKA, R. and ERDELYI, M. (2001). An interaction type of genetic screen reveals a role of the Rab11 gene in oskar mRNA localization in the developing Drosophila melanogaster oocyte. Genetics 158:1177-1788.

LAKHOTIA, S.C. and TAPADIA, M.G. (1998). Genetic mapping of the amide response element(s) of the hsr-omega locus of Drosophila melanogaster. Chromosoma 107:127-135.

PELISSIER, A., CHAUVIN, J.P. and LECUIT, T. (2003) Trafficking through Rab11 endosomes is required for cellularization during Drosophila embryogenesis. Curr.
Biol. 13:1848-1857.

PEREIRA-LEAL, J.B. and SEABRA, M.C. (2001). Evolution of the Rab family of small GTP-binding proteins. J. Mol. Biol. 313:889-901.

PIRROTTA, V. (1986). Cloning Drosophila genes. In: Drosophila, A practical approach (ed. Roberts, D.R.), IRL Press, Oxford, pp.83-110.

PIRROTTA, V. (1988). Vectors for P-mediated transformation in Drosophila. Biotechnology 10:437-456.

READY, D.F., HANSON, T.E. and BENZER, S. (1976). Development of the Drosophila retina, a neurocrystalline lattice. Dev. Biol. 53:217-240.

RIGGS, B., ROTHWELL, W., MISCHE, S., HICKSON, G.R., MATHESON, J., HAYS, T.S., GOULD, G.W. and SULLIVAN, W. (2003). Actin cytoskeleton remodeling during early Drosophila furrow formation requires recycling endosomal components Nuclear-fallout and Rab11. J. Cell Biol. 163:143-154.

ROBERTSON, H.M., PRESTON, C.R., PHILLIS, R.W., JOHNSON-SCHLITZ, D.M., BENZ, W.K. and ENGELS, W.R. (1988). A stable genomic source of $P$ element transposase in Drosophila melanogaster. Genetics 118:461-470.

RUBIN, G.M. and SPRADLING, A.C. (1982). Genetic transformation of Drosophila with transposable element vectors. Science 218:348-353.

SATOH, A.K., O'TOUSA, J.E., OZAKI, K. and READY, D.F. (2005). Rab11 mediates post-Golgi trafficking of rhodopsin to the photosensitive apical membrane of Drosophila photoreceptors. Development 132:1487-1497.

SATOH, A.K., TOKUNAGA, F., KAWAMURA, S. and OZAKI, K. (1997). In situ inhibition of vesicle transport and protein processing in the dominant negative Rab1 mutant of Drosophila. J. Cell Sci. 110:2943-2953.

SHETTY, K.M., KURADA, P. and O'TOUSA, J.E. (1998). Rab6 regulation of rhodopsin transport in Drosophila. J. Biol. Chem. 273:20425-20430.

SPRADLING, A.C. and RUBIN, G.M. (1982). Transposition of cloned P elements into Drosophila germ line chromosomes. Science 218:341-347.

STOWERS, R.S. and SCHWARZ, T.L. (1999). A genetic method for generating Drosophila eyes composed exclusively of mitotic clones of a single genotype. Genetics 152:1631-1639.

URBE, S., HUBER, L.A., ZERIAL, M., TOOZE, S.A. and PARTON, R.G. (1993). Rab11, a small GTPase associated with both constitutive and regulated secretory pathways in PC12 cells. FEBS Lett 334:175-82.

WANG, X., KUMAR, R., NAVARRE, J., CASANOVA, J.E. and GOLDENRING, J.R. (2000). Regulation of vesicle trafficking in madin-darby canine kidney cells by Rab11a and Rab25. J Biol Chem 275:29138-29146.

XU, T. and RUBIN, G.M. (1993). Analysis of genetic mosaics in developing and adult Drosophila tissues. Development 117:1223-1237.

ZERIAL, M. and MCBRIDE, H. (2001). Rab proteins as membrane organizers. Nat. Rev. Mol. Cell Biol. 2:107-117.

Received: February 2005

Reviewed by Referees: May 2005

Modified by Authors and Accepted for Publication: August 2005 